\title{
Contents, Vol. 29, 1966
}
H. CHIARI, Wien
H. COTTIER, Bern
H. FEY, Bern
J. FIRKET, Liège
C. HALLAUER, Bern
J. LINDENMANN, Zurich
COLLABORATORES

A. L. OLITZKI, Jerusalem

R. H. REGAMEY, Geneve

F. C. ROULET, Basel

E. RUTISHAUSER, Geneve

R. SILBERBERG, Saint Louis, Mo.

H. SMETANA, Washington R. TULASNE, Strasbourg E. UEHLINGER, Zurich M. WELSCH, Liège A.WERTHEMANN, Basel

REDACTORES A. GRUMBACH, Zurich J. R. RÜTTNER, Zurich

Vol. 29

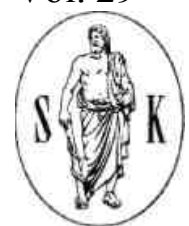

1966

\section{BASEL (Schweiz) S. KARGER NEW YORK}

Index

Adamiker, D., Breitfellner, G. und Neuhold, R.:

Zur Ultrastruktur der Staphylokokkenphagen, des Befallsmodus und der Veränderungen der Staphylokokken nach Phagenbefall .... 489

Angervall, L., Dahl, I. and Säve-Söderbergh, J.:

The Aortic Vasa vasorum in Juvenile Diabetes

Angyal, T.: $\quad$ vide Rauss, $\mathrm{K}$.

Babala, J.: vide Lesko, A.

Babusceac, Livia: vide Diosi, P.

Bachofen, R. und Heer, Elisabeth:

Über die Synthese von Alanin und Aspartat aus Acetat und C02 bei

Clostridium butyricum 747

Bachofen, R.: vide Bolliger, E.

Bairati, A.: vide Pernis, B.

Banic, S.: $\quad$ A New Liquid Medium for the Primary Cultivation of Tubercle

Bacilli 243 
Barber, Cella, Lazar, I. et Meitert, Eugenie:

Contribution à $\Gamma$ étude des relations entre corynébactéries diphthériques et corynébactéries phytopathogènes. La spécificité sérologique des polysaccharides isolés des corynébactéries phytopathogènes . . 84 Barber, C, Meitert, E., Bica-Pop $\pi$, V. et Saragea, A.:

Sur les relations immunochimiques des corynébactéries animales avec Corynebacterium diphtheriae et les corynébactéries phytopathogènes 377

Barber, C, Pleceas, P. et Olinesco, E.:

Contribution à $\Gamma$ étude antigénique des streptocoques du Groupe D

Lancefield. Les antigènes de groupe et de type de Streptococcus equinus $\quad 480$

BÁRTA, K.: Zur Frage der antigenwirksamen Komponenten der Lunge bei der

Pneumocysten-Pneumonie 63

Baumgartner, H.-R. und Studer, A.:

Folgen des Gefäßkatheterismus am normo- und hypercholesterin-

ämischen Kaninchen 393

Baumgartner, H.: vide Kästli, P.

Becht, H.: Untersuchungen über das serologische Verhalten eines Säureex-

traktes aus Brucellen mit besonderer Berücksichtigung der indirekten

Hämagglutination 204

Beck, G.: $\quad$ vide Rudat, K.-D.

Becla, E.: $\quad$ vide Kryñski, S.

Bica-Popii, V.: vide Barber, C.

Bolliger, E., Rast, Dora und Bachofen, R.:

Synthese von Aminosäuren in Extrakten von Agaricus bisporus . . 742

Bonifas, V. H.: Un variant de Mycoplasma pneumoniae $\quad 562$

Boss, J. H.: vide Weinberger, N. J.

Böszörményi, J.: vide Kocsár, L. Bourne, G. H.: vide Sharma, N. N. Breitfellner, G., Lunglmayr, G. und Neuhold, R.:

Histochemische und elektronenmikroskopische Untersuchungen zur

Wirkung von Ajmalin am Meerschweinchenherzen 414

Breitpellner, G.: vide Adamiker, D. Bucher, O. und Riedel, B.:

Degeneration und Regeneration von Fibrocytenkulturen nach

Tintenstiftverletzungen $\quad 176$

Bugalski, R.: vide Kryñski, S.

Buitenweg, D. W.: vide Hooff, A. van den Cinátl, J. and Marhoul, Z.:

Structural Changes in Aging Serum (Part I) 459

Cinátl, J., Liebl, V., Hurych, J., Tupý, J., Michalec, C. and Kourílek, K.:

Structures Occuring in Aging Serum (Part II) 470

Costin, I. D.: Diarrheal Diseases in Children and Adults Associated with Escheri-

chia coli Strains not Belonging to Enteropathogenic Serotypes. . 214

Dabx, I.: $\quad$ vide Angervall, L.

Denk, H., Flamm, H. und Kovac, W.:

Histologische Untersuchung von graviden Mäusen nach Infektion

mit Listeria monocytogenes 163

Diosi, P., Babusceac, Livia, Nevinglovschi, Olimpia and Stoicanescu, Ann: 
Duration of Cytomegaloviruria in Nursery Children 513

Doodewaard, J.: vide Wikén, T. 0.

Engelbrecht, E.: Test rapide pour le diagnostic de la syphilis avec un antigène tréponémique 553

Elsden, S. R.: The Contribution of the Ruminant to Microbiology 602

Fiechter, A. und Meyenburg, K. von :

Wachstum und Gasstoffwechsel von Saccharomyces cerevisiae in kontinuierlicher Kultur 696

Flamm, H.: vide Denk, H.

Frank, W.: $\quad$ vide Rudat, K.-D.

Fuchs, U.: $\quad$ Kristalle in Blutungs- und Nekroseherden der Rattenlunge .... 8

Garcia, J. M.: vide Regamey, R. H.

Gartner, J.: "Long-spacing" Kollagen in der Wand einer Netzhautarteriole ... 55

Gaschen, M.: Etude in vitro d'une nouvelle preparation destinée à la désinfection

remanente des mains 811

Gehriger, G.: ̈̈ber die Gefriertrockung von käsereitechnisch wichtigen Milchsäure-

bakterien 639

Geneidi, A.: vide Sharaf, A.

Gindrat, J. J.: vide Regamey, R. H.

Grumbach, A.:25 Jahre Schweizerische Mikrobiologische Gesellschaft - 25 Jahre

Mikrobiologie 663

Gruner, J.: $\quad$ Untersuchungen zur Technik der Sauerstoffversorgung bei Sub-

mersfermentationen 767

Guggenheim, B., König, K. G. und Mühlemann, H. R.:

Der Rattenversuch in relativer Gnotobiose als Test für die Kario-

genität von Streptokokken 656

Hahlweg, Gertrud und Orf, G.:

Sogenannte fibroplastische Myocarditis bei Oxalose

Hasler, Mary: vide Silberberg, M. Heer, Elisabeth: vide Bachofen, R. Heyden, S. und Rüttner, J.

R.:

Die Beeinflussung des Cholesterinspiegels durch Coffein im Tierver-

such 291

Hiller, Chr.: Kautschukparaffin - ein vorzügliches Einbettungsmedium zur

Herstellung histologischer Serienschnitte 276

Hoof, A. van den, Nie, C. J. van, and Buitenweg, D. W.:

Histology and Polysaccharide Histochemistry of Heterotopic Bone

Formation

17

Hurych, J.: vide Cinátl, J.

Jeljaszewicz, J.: vide Szmigielski, S.

Jobbágy, A.: vide Király, K.

Kalowski, Miron : On the Usefulness of Anaerobic Glucose and Mannitol Decomposition in the Classification of Micrococci (Family Micrococcaceae) .... 126 Kästli, P., Baumgartner, H. und Rüttimann, G.:

Die Vermehrung verschiedener Bakterienarten in Milch mit erhöhtem

Zellgehalt $\quad 630$

Kedzia, W., Musielak, M., Kedzia, B., Koniar, H. and Pniewska, E.: 
Enzymatic Activity of Coagulase-positive Staphylococcus aureus

Strains Isolated from Patients and Healthy Carriers 307

Kedzia, B.: vide Kedzia, W.

Kétyi, I.: $\quad$ vide Rauss, $\mathrm{K}$.

Király, K.: $\quad$ Quantitative Fluorescent Treponemal Antibody (FTA) Studies with

Balanitis and "Problem" Sera 75

Kirlly, K. and Jobbágy, A.:

Control of Conjugation of Fluorescent Protein Tracing 865

Knetemann, J. C. A.: vide Wikén, T. O. Kocsár, L., Böszörményi, J. and Virág, S.:

Immunoresponse of Mature Rabbits and Guinea-Pigs Having

Developed an Additional Thymes 156

Koniar, H.: $\quad$ vide Kedzia, W.

König, H.: $\quad$ vide Nicolet, J.

König, K. G.: vide Guggenheim, B.

Kourílek, K.: vide Cinátl, J.

Kovac, W.: vide Denk, H.

Krbavcic, A.: vide Schauer, P.

Krech, U.: Antikörper-Reaktionen bei Infektionen mit dem Mycoplasma pneu-

moniae 558

Kruml, J.: $\quad$ vide Trnka, L.

Krynski, S., Becla, E., Bugalski, R., Podhajska, Anna and Samet, A.:

Staphylococcus aureus Types Isolated from Hospital Sources and

from Healthy Carriers 854

Kuska, J.: $\quad$ vide Trnka, L.

Lazar, I.: $\quad$ vide Barber, Cella

Leisinger, Th. : Enzymatische Untersuchungen am Wildtyp und an einer cellulosefreien Mutante von Acetobacter xylinus $\quad 756$

Index autorum ad Vol. XXIX

$(B)=$ Buchbesprechungen - Livres nouveaux - Book reviews $(V)=$ Vortrag - Communication Report

Angaben in Klammern ( ): Die Arbeit ist nicht (resp. nicht vollständig) erschie-nen oder wird an einer ande $\Gamma$ en Stelle in extenso publiziert werden. - Indications entre parentheses ( ) : Le travail n'a pas paru (ou en partie) ou sera publié ailleurs in extenso. - Notes in parentheses (): The paper has not been published (or in part only) or will be published in extenso elsewhere.

Adamiker, D., Breitfellner, G. und

Neuhold, R., 489 Angervall, L., Dahl, I. and Säve-

Söderbergh, J., 431 Angyal, T., v. Rauss, K.

Babala, J., v. Lesko, A.

Babusceac, L., v. Diosi, P.

Bachofen, R., v. Bolliger, E.

Bachofen, R. und Heer, E., 747 (V)

Bacq, Z. M. (Editor), 387 (B)

Bader, G., 874 (B)

Bairati, A., v. Pernis, B.

Bajusz, E., 520 (B)

Banic, S., 243 
Barandun, S., v. Koblet, H.

Barber, C., Lazar, I. et Meitert, E., 84

Barber, C., Meitert, E., Bica-Popii, V.

et Saragea, A., 377 Barber, C., Pleceas, P. et Olinesco,

E., 480 Bárta, K., 63

Baumgartner, H., v. Kästli, P. Baumgartner, H.-R. und Studer, A.,

393 Becht, H., 204 Beck, G., v. Rudat, K.-D. Becla, E., v. Kryฑ́ski, S. Beerens, H. et Tahon-

Castel, M.,

389 (B) Beeuwkes, A. M., 133 (B) Bica-Popii, V., v. Barber, C. Bloch, H., 258 (B) Bönicke, R.,

v. Freerksen, E. Böszörményi, J., v. Kocsár, L. Bolliger, E., Rast, D. und Bachofen,

R., 742 (V) Bonifas, V. H., 562 (V) Boroviczény, v. de Boroviczény Boss, J. H., v. Weinberger, N. J. Bourne, G. H., 133 (B)

Bourne, G. H., v. Sharma, N. N. Breitfellner, G., v. Adamiker, D. Breitfellner, G., Lunglmayr, G. und

Neuhold, R., 414 Bucher, 0. und Riedel, B., 176 Bürki, F., 875 (B),879 (B) Bugalski, R., v.

Kryү́ski, S. Buitenweg, D. W., v. Van den Hooff,

A. Buschmann, O., v. Willich, E.

Cinátl, J. and Marhoul, Z., 459 Cinátl, J., Liebl, V., Hurych, J.,

Tupý, J., Michalec, C, and

Kouiílek, K, 470 Constantinides, P., 390 (B) Gossel, L., 388 (B) Costin, I. D., 214 Cowan, S. T.

and Steel, K. J., 387 (B)

Dahl, I., v. Angervall, L.

De Boroviczény, Ch. G., 392 (B)

Denk, H., Flamm, H. und Kovac, W.,

163 Deucher, F., Kappert, A., Miller, G.

und Rudler, J.-C. (Editores),

784 (B) Diggelmann, H., v. Koblet, H. Diosi, P., Babusceac, L., Neving-

lovschi, O. and Stoicanescu, A., 513 Doodewaard, J., v. Wikén, T. O.

Eggen, R. R., 878 (B) Egli, M., 260 (B) Elsden, S. R., 602 (V) Engelbrecht, E., 553 (V)

Fabry von Hilden, W., 391 (B) Fenner, F. and Ratcliffe, F. N.,

879 (B)

Lesko, A., Babala, J. and Lojda, Z.:

Early Histological and Histochemical Changes Produced in the

Arterial Wall of Dogs by Injecting Blood Clot 279

Liebl, V.: vide Cinátl, J.

Likar, M.: $\quad$ vide Schauer, P.

Lindt, S.: $\quad$ vide Nicolet, J.

Lojda, Z.: $\quad$ vide Lesko, A.

Lorez, H.-P.: vide Studer, A.

Lorez, H. P.: vide Studer, A.

Lunglmayr, G.: vide Breitfellner, G.

Lupulescu, A., Potorac, E., Vasilescu, T., Pop, A., Merculiev, E., Opri§an, R. and

Oprißan, A.: Effects of the Anabolic Steroid (4-Chlorotestosterone) on Antibody

Production in Hyper- and Hypothyroid Rabbits $\quad 800$ 
Mannheim, W.: Limitierende Faktoren in Mischkulturen eines obligaten Aerobiers (Achromobacter metalcaligenes) mit Haemophilus influenzae resp.

mit Anaerobiern 341

Marhoul, Z.: vide Cinátl, J.

Meitert, E.: $\quad$ vide Barber, $C$.

Meitert, Eugenie : vide Barber, Cella

Merculiev, E.: vide Lupulescu, A.

Meyenburg, K. von : vide Fiechter, A.

Michalec, C.: vide Cinátl, J.

Mierzejewska, I.: vide Parnas, J.

Milanesi, S.: vide Pernis, B.

Modde, H.: $\quad$ In vitro-Prüfung der Stabilität halbsynthetischer Penicilline in

Frauenmilch 451

Modde, H. und Riepl, G.:

Quantitative Pruning der Chloramphenícol-Empfindlichkeit von

Typhus- und Paratyphus-Salmonellen $\quad 616$

Mrugowsky, G.: vide Rudat, K.-D.

Muftic, M. and Schroder, E.:

Peptidasegram in the Differentiation of Bacilli Species 252

Mühlemann, H. R.: vide Guggenheim, B.

Müller, E.: $\quad$ vide Scholer, J.

Musielak, M.: vide Kedzia, W.

Negm, S.: $\quad$ vide Sharaf, A.

Neuhold, R.: vide Adamiker, D.

Neuhold, R.: vide Breitfellner, G.

Nevinglovschi, Olimpia: vide Diosi, P.

Nicolet, J. und König, H.:

Zur Haemophilus-Pleuropneumonie beim Schwein. Bakteriologische, pathologisch-anatomische und histologische Befunde (Vorläufige

Mitteilung) 301

Nicolet, J., Lindt, S. et Scholer, H. J.:

L'avortement mycosique de la vache. Considerations sur le diag

nostic de routine 644

Nie, C. J. van: vide Hooff, A. van den

Nievelt, M. C. A., van: vide Wikjén, T. 0.

Olinesco, E.: vide Barber, C.

Oprißan, A.: vide Lupulescu, A.

Opbißan, R.: vide Lupulescu, A.

Obf, G.: $\quad$ vide Hahlweg, Gertrud

Parnas, J., Tuszkiewicz, M., Mierzejewska, I. und Szunke, B.:

Weitere immunologische Analyse der Brucellaallergene 438

Pernis, B., Bairati, A. and Milanesi, S.:

Cellular and Humoral Reactions to Freund's Adjuvant in Guinea-Pigs 837

Piguet, J. D.: Lysotypie staphylococcique. Frequence du type 29 dans 1'hospitalisme 193

Piguet, J. D.: Lysotopie staphylococcique et sensibilité aux antibiotiques. Résultats 
interprétés à Tordinateur électronique

Pleceas, P.: $\quad$ vide Barber, C.

Pniewska, E.: vide Kedzia, W.

Podhajska, Anna: vide Kryñski, S.

Pop, A.: $\quad$ vide Lupulescu, A.

Potorac, E.: vide Lupulescu, A.

Rast, Dora: vide Bolliger, E.

Rauss, K., Kétyi, I. and Angyal, T.:

Experimental Shigellosis in Mice

Regamey, R. H., Gindrat, J. J. et Wantz, M.:

Quelques aspects de la toxinogenèse tétanique

Regamey, R. H. et Garcia, J. M.:

Problèmes poses par Thospitalisme dans un service de chirurgie . . 543

Riedel, B.: $\quad$ vide Bucher, 0.

Riepl, G.: $\quad$ vide Modde, $\mathrm{H}$.

Rosse, W. F.: vide Swarm, R. L.

Rudat, K.-D., Beck, G., Frank, W. und Mrugowsky, G.:

Über das Vorkommen von Salmonellen bei Reptilien in Zoologischen

Garten. Vorläufige Mitteilung über Untersuchungen in den Zoolo

gischen Garten Basel, Bern und Zurich

623

Rüttimann, G.: vide Kästli, P.

Rüttner, J. R.: vide Heyden, S.

Samet, A.: $\quad$ vide Kryñski, S.

Saragea, A.: vide Barber, C.

Säve-Söderbebgh, J.: vide Angebvall, L.

Schaueb, P., Likab, M., Tisler, M. and Krbavcic, A.:

Studies of Some Substances with Antiviral Activity. III. The

Inhibition of Poliovirus Type 1 and 2 Induced Cytopathogenic

Changes in Cell Cultures of Human Embryonic Kidneys by Deriva

tives of Thiazolidine Acetic Acid 506

Scheffebs, W. A.: vide Wikén, T. 0.

Scholer, H. J. und Müller, E.:

Beziehungen zwischen biochemischer Leistung und Morphologie bei

Pilzen aus der Famile der Mucoraceen $\quad 730$

Scholer, H. J.: vide Nicolet, J.

Schroder, E.: vide Muftic, M.

Schwarz, P.: A Method for Establishing Buffer Mixtures by Means of the Serial

Dilution Method Ill

Sharaf, A., Geneidi, A. and Negm, S.:

Further Study on the Antibacterial Effect of H. sabdariffa .... 120

Shabma, N. N. and Bourne, G. H. (Atlanta, Ga.):

Histochemical Demonstration of DNP-Linked Dehydrogenases in

Trichomonas vaginalis 366

Silberberg, M., Hasler, Mary and Silbebberg, Ruth:

Articular Cartilage of Dwarf Mice: Submicroscopic Effects of

Somatotrophin 137 
Silbebbebg, Ruth : vide Silbebberg, M.

Stenzel, W.: Uber die Beziehungen zwischen Allgemein- und Schleimhautpathogenität der Shigellen 236

Stoicanescu, Ann : vide Diosi, P.

Stboun, J.: Précocité de la souffrance hépatocytaire et de Thyperplasie régéné-

ratrice lors d'obstruction biliaire extra-hépatique. Etude expéri-

mentale et clinique 265

Studer, A. und Lobez, H. P.:

Zum Mechanismus der thrombinínduzierten Fibrinbildung an der

geschädigten Kaninchenaorta 285

Studeb, A. und Lobez, H.-P.:

Experimentelle Untersuchungen über die Bedeutung vorbestehender

Gefäßveränderungen für die Anlagerung von Fibrin 406

Studeb, A.: $\quad$ vide Baumgabtneb, H.-R.

Swabm, R. L., Waldmann, T. A. and Rosse, W. F.:

Polycythemia in Rats with Adrenal Cortical Carcinoma Transplants 29

Szmigielski, S., Jeljaszewicz, J. and Wilczynski, J.:

5'-Nucleotidase Activity in Rabbit Leukocytes during Experimental

Infections 186

Szunke, B.: $\quad$ vide Parnas, J.

Theileb, K.: Gibt es eine Vakzine-Virusembryopathie? Versuche mit besonderer

Berücksichtigung verschiedener Variabeln im Säugetierexperiment. 825

Tisleb, M.: vide Schaueb, P.

Tbnka, L., Kbuml, J., Kuska, J. und Ubbancik, R.:

Zur Frage der kavernösen Modelltuberkulose bei Kaninchen .... 228

Tupý, J.: $\quad$ vide Cinátl, J.

Tubian, G. et Viswanathan, M. A.:

Facteurs nucléo-cytoplasmiques et différenciation sexuelle des AUomyces. Inhibitions sélectives par les acridines et $\Gamma$ actinomycine D 705

Tuszkiewicz, M.: vide Parnas, J.

Ubbancik, R.: vide Trnka, L.

Vasilescu, T.: vide Lupulescu, A.

Virág, S.: $\quad$ vide Kocsár, L.

Viswanathan, M. A.: vide Turian, G.

Waldmann, T. A.: vide Swarm, R. L.

Wanner, A.: Experimentelle Aortalipoidose und renale Hypertonie der Ratte:

Die Bedeutung der Schilddrüsen- und Gonadenfunktion 785

Wantz, M.: vide Regamey, R. H.

Weinbebger, N. J. and Boss, J. H.:

A Comparative Study of Nephrotoxic Serum Antigens of Diverse Rat

Organs 324

Welsch, M.: Baçtériolyse et parois cellulaires bactériennes 571

Wettstein, P. U.: Quantitative Staubuntersuchungen an Süikoselungen schweizerischer

Provenienz 39

Wikén, T. 0., Nievelt, M. C. A., van, Knetemann, J. C. A., Doodewaahd, J. and 
Scheffehs, W. A.: The Influence of Free Oxygen at High Concentrations on the Rate of Alcoholic Fermentation in a Yeast Showing a Negative Pasteur

Effect in Succinic Acid-Succinate Buffer 676

Wilczynski, J.: vide Szmigielski, S.

Yang, Y. H.: The Multicentric Renal Adenocarcinomas in situ of a Dog .... 181

Vekhandlungsberichte - Proceedings - Congrès

Schweizerische Mikrobiologische Gesellschaft, 25. Jahresversamm-lung, 18. bis 19. Juni 1966, in Winterthur - Société Suisse de Micro-biologie, 25e assemblée annuelle, du 18 au 19 juin 1966, à Winterthur 521

Mitgliederliste der Schweizerischen Mikrobiologischen Gesellschaft -

Membres de la Société Suisse de Microbiologie 776

Buchbesprechungen - Book Reviews - Livres Nouveaux . . 133, 257, 387, 519, 784, 873

RICHTIGSTELLUNG 873

Varia 392, 883

Index Rerum 884

Index Autorum 901

Alle Rechte, insbesondere das der Übersetzung in fremde Sprachen, vorbehalten.

Ohne ausd $\mathrm{ückliche}$ Genehmigung des Verlages ist es auch nicht gestattet, dieses Buch oder

Teile daraus auf photomechanischem Wege (Photokopie, Mikrokopie) zu vervielfältigen.

(C)

Copyright 1.966 by S. Karger AG., Basel Printed in Switzerland by Buchdruckerei Friedrich Reinhardt AG., Basel Cliches: Aberegg-Steiner \& Cie. AG., Bern, und Steiner \& Cie. AG., Basel 